cooling, and it is demonstrated that cooling leads to instability and fragmentation of the dense disk that is formed. This disruptive fragmentation is not seen for purely isothermal calculations unless a very low-isothermal temperature is picked. I would caution against interpreting pure isothermal calculations. Cooling should be included (Hunter,Sandford, Whitaker and Klein; Astrophys. J. 1986).

\title{
ON EARLY EVOLUTION OF ACCRETING STARS
}

\author{
B.M. Shustov, A.V. Tutukov \\ Astronomical Council of the USSR Academy of Sciences, \\ Moscow, USSR
}

Accretion is a dominant factor in the early evolution of stars. The first time an accretion regime settles in is when a dusty opaque core forms. The mass of adiabatically contracting core inside the isothermally collapsing envelope depends only on the optical properties of dust. Spherically symmetric models of dusty cores were constructed using the Henyey technique with accretion boundary conditions (Menshchikov 1986). It appears that all protostars with normal chemical composition should pass through the stage of a quasistatic dusty core. The evolution of dusty cores is similar to that of "normal" young stars with accretion. One could distinguish convective, radiative and central core contraction phases. The life-time $t_{c}$ of the core depends on the core mass $M_{C}$ and the accretion rate $\dot{M}$ (for $M_{C}=0.01 M_{\odot}$ and $\dot{M}=1.6 \times 10^{-6}, 1.6 \times 10^{-5} \mathrm{M}_{\odot} /$ year $t_{c}=1.2 \times 10^{4}, 3 \times 10^{3}$ yrs consequent1y). After dust exhaustion in the core it collapses and a central ionized quasistatic region grows in several tens of years. A flash of infrared radiation at the moment is not excluded.

According to numerical models (Tutukov and Shustov 1981) $\dot{M}$ is nearly constant during the main accretion phase (time scale $t_{a}$ (yrs) $\sim 3 \times 10^{5} \mathrm{M} / \mathrm{M}_{\odot}$ for a cold cloud) and it stops abruptly in a time shorter than $t_{a}$. This abrupt cessation of accretion is one of the reasons for the so-called "birthline" in the H-R diagram.

The evolution of accreting stars $\left(0.1<\mathrm{M} / \mathrm{M}_{\odot}<6.0\right)$ assuming constant $\dot{M}=3 \times 10^{-6}$ and $10^{-5} \mathrm{M}_{\odot}$ /year was calculated by Tutukov and Chiefi (1985). The major problem concerns the fate of the energy liberated at the shock front. Two limiting cases are: a) almost all the energy liberated at the shock front is lost outward and the accreted matter therefore has low entropy, b) almost all the energy goes into the star. Evolutionary tracks of accreting stars (solid lines), tracks after cessation of accretion (dashed lines) and the "birthline" (zigzag line) are shown in Figure 1. The location of the tracks for models with accretion is insensitive to the exact value of $\dot{M}$. More important is the relation between $t_{a}$ and the thermal time scale $t_{\mathrm{KH}}$. The turnover point in the tracks for case $\mathrm{b}$ ) means that $t_{K H}$ becomes smaller than $t_{a}$. 


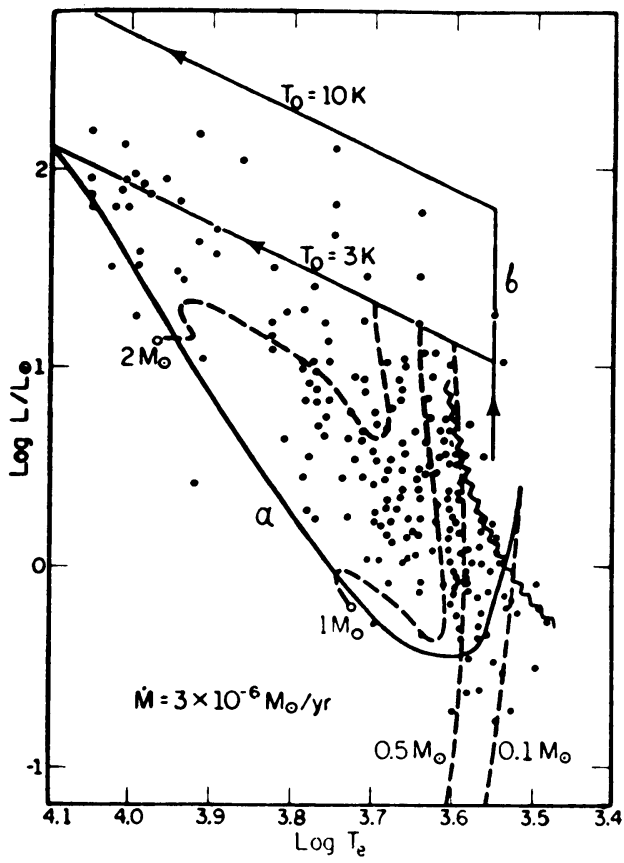

A comparison between the calculated evolutionary tracks and the positions of young stellar members of several open clusters (the dots in Figure 1 are taken from Cohen and Kuhi 1979) shows that the accretion of low entropy matter cannot explain the observed distribution of these stars. To obtain an agreement with the observations one has to assume that a large fraction of the gravitational energy liberated at the shock front goes to increase the heat content of the stars. For low mass stars the relaxation of their overheated upper layers, after an abrupt cessation of accretion, may be a source of energy for activity of young stars. These stars can release the excess of energy in some nonstationary manner.

\section{REFERENCES}

Cohen, M., Kuhi, L.V.: 1979, Astrophys. J. Supp1. 41, 743. Menshchikov, A.B.: 1986, Nauchn. Inform. 62, in press. Tutukov, A.V., Chiefi, A.: 1985, Nauchn. Inform. 58, 40. Tutukov, A.V., Shustov, B.M.: 1981, Astron. Zh. 58, 109.

MOUSCHOVIAS: Does the sudden end of accretion occur because you run out of mass or because of some (what?) outward force generated by the central object?

SHUSTOV: For low mass stars the reason for accretion to stop is the exhaustion of matter in a given fragment. The stellar wind switches on when practically all the mass is accumulated in the star. For a more massive protostar there are effective mechanisms of cessarion of accretion: radiative pressure on dust, shock wave propagation induced by ionization and the stellar wind. 